\title{
Oral Systemic Data Driven Computation Link
}

Science and technology tools specially with emergence of internet based real time computing systems expanded the analytic ability of data analysis at large. This is also refereed as "Big Data Analysis" where data is enormously huge and higher application in analytical analysis. The big data is basically classified by either volume, velocity, variety, veracity and value. With big data analytics, we can ultimately fuel better and faster decision support tool that enhance ability for better application in all business models including in the field of dentistry. Dentistry has changed a great deal in areas such as diagnosis, prevention and treatment over the last three decades. The role of the dentist has significantly changed over the last decade, and the dentist's role and responsibility are now to maintain overall health rather than focus on oral health only. The interaction between oral and systemic health are bidirectional and complex, involving many pathways. Recent evidence suggests that periodontal infection

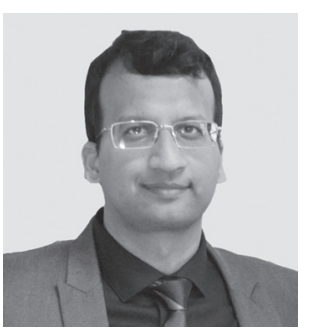

may significantly enhance the risk for certain systemic diseases or alter the natural course of systemic conditions; and conditions in which influences of periodontal infection are documented include coronary heart diseases (CHD) and CHD-related events such as angina and infarction, atherosclerosis, stroke, diabetes mellitus; preterm labor, low-birth-weight delivery; and respiratory conditions such as chronic obstructive pulmonary diseases, etc.

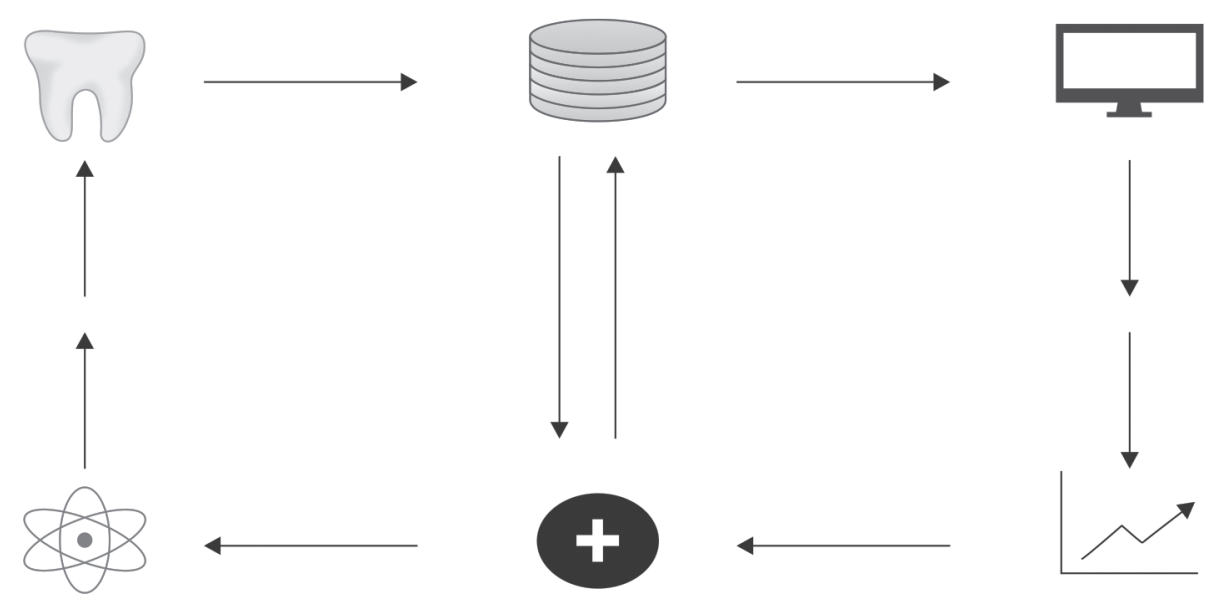

Data driven dentistry will play a major role not only on electronic health records but also on better understanding in operability of oral-systemic linkage in a way of organized dentistry. Integrating methods from the patient history, disease pattern, treatment and treatment outcome to assist in design computer applications, implement system and evaluation system in the management of healthcare information. It is a rapidly growing field of knowledge that comprehends a number of zones from data processing and machine learning to statistics for application in the dental sciences. Data driven dentistry have the potential to transfigure dental industry through the innovation of new looms to data organization in dental research, patient treatment and particularly prevention of oral disease. More knowledge and significant deep data mining are much more needed to fully understand its magnitude and potential applicability.

Rajiv Saini Editor-in-Chief 